\title{
Surface modification of PDMS based microfluidic systems by tensides
}

\author{
E. Holczer ${ }^{1,2, a}$, Z. Fekete ${ }^{1,2, b}$, P. Fürjes ${ }^{1, c}$ \\ ${ }^{1}$ Research Centre for Natural Sciences, MEMS Laboratory - MTA TTK MFA MEMS \\ H-1525 Budapest P.O.Box 49. \\ ${ }^{2}$ Budapest University of Technology and Economics, H-1111 Budapest, Budafoki str. 8. \\ aholczer.eszter@ttk.mta.hu (corresponding author), \\ bfekete.zoltan@ttk.mta.hu, ćlurjes.peter@ttk.mta.hu
}

Keywords: PDMS, surface modification, tenside, microfluidics

\begin{abstract}
The material aspects of a polymer based microfluidic structure were characterised considering the compatibility of the system with bioanalytical applications. The polydimethylsiloxane (PDMS) based channel system is to be integrated in a full polymer photonic biosensor device developed within the European Union project P3SENS (FP7-ICT4-248304).

This work is intended to define a modified material composition, which is appropriate to improve both the wettability and the non-specific protein binding characteristics of the PDMS significantly. Triton X-100 (Sigma-Aldrich) surfactant was added to the raw PDMS before polymerisation. The influence of the tenside was studied considering the polymerisation reaction, the surface characteristics and the functional applicability. To test the hydrodynamic behaviour and non-specific protein adsorption on the surfaces, phosphate buffered saline (PBS) solution and fluorescent labelled human serum albumin (HSA) was applied in a microfluidic capillary system.
\end{abstract}

\section{Introduction}

The main aim of the development is to realise robust bioanalytical device for recognition specific proteins in human blood referring to cerebral vascular accident, e.g. stroke. Integrated microfluidic elements are essential components of all lab-on-a-chip devices. The proposed microfluidic system must provide reliable sample transport avoiding the deterioration of device sensitivity. On the other hand, some simple sample preparation functions, such as washing, mixing or dilution is to be included as well. As structural material, PDMS was selected due to its comfortable and cost-effective fabrication technique by Rapid Prototyping.

The PDMS is a silicon based organic polymer: $\left(\mathrm{H}_{3} \mathrm{C}\right)_{3}\left[\mathrm{Si}\left(\mathrm{CH}_{3}\right)_{2} \mathrm{O}\right]_{\mathrm{n}} \mathrm{Si}\left(\mathrm{CH}_{3}\right)_{3}$ which is absolutely feasible to form microfluidic structures in (bio)analytical applications due to its reliable geometric transfer, flexibility, transparency, biocompatibility, chemical and biological resistance and price, moreover it is beneficial for large scale production by molding SU-8 or Si replicas [1]. Apparent drawback of the material is the hydrophobic surface characteristics, significantly affecting the maximal flow rate in the channel system. Besides, the non-specific binding of proteins or ligands on the channel surfaces is also a critical issue due to the possible depletion of the target molecules during the transport to the active sensing area of the device. The possible long term degradation is also crucial.

Considering the requirements of complex sample preparation systems our objective is to develop polymer based microfluidic system applying transport channels with low hydrodynamic resistance and low non-specific protein binding on the surfaces. The adequate modification of the PDMS could simultaneously improve both deficiencies of the structural material, resulting high throughput microfluidic sample preparation system. Additional advantage of the modified material composition could be the possible application to passive microfluidic capillary pump system. 


\section{Surface modification techniques}

In order to modify surface characteristics of the PDMS, several different techniques like oxidation, grafting and embedding were reported [2]. The oxidation process can be implemented by air oxidation, oxygen plasma [3] or oxidizing solvents [4]. During these processes hydrophilic silanol $(\mathrm{Si}-\mathrm{OH})$ and silicon-dioxide $\left(\mathrm{SiO}_{2}\right)$ groups are formed on the surface [5]. Disadvantage of these methods is that the surface becomes hydrophobic again quickly due to the shorter, delocalized monomer chains of the PDMS freely moving in the polymer matrix [6]. Eventually, fast decrease in contact angle can be observed resulting significant improvement in the surface wetting [7], however, the applicability of the PDMS is still critical regarding long-term stability. Another possible surface activation method can be the silane chemistry, applying Amino-Silanes as 3Aminopropyltriethoxysilane (APTES) [8] or Hexamethyldisilazane (HMDS). In our case surfactants were added into the PDMS before polymerization to modify the characteristics of the material [9]. This technique is expected to create stable hydrophilic layer on the PDMS surface through the general modification of the bulk material. When the free surfaces of the PDMS contact with aqueous solutions, the tenside chains build up oriented monolayer on the polymer-liquid interface due to the unobstructed diffusion of the embedding molecules in the bulk matrix, and result more hydrophilic characteristics on the surface. Surfactant molecules do not affect the conventional bonding technology of PDMS substrates, the strong adhesion between PDMS and other suitable materials like glass or silicon could be easily developed before the substrates contact with aqueous solutions.

\section{Realisation of PDMS-based microfluidic channel system}

The polymer based microfluidic structure was realised by fast prototyping applying SU-8 epoxy based negative photoresist as replica for PDMS moulding. An improved 3D multilayer formation process was developed in order to achieve reliable SU-8 masters involving high aspect ratio sidewalls and advanced functional elements of the microfluidic system. Fabrication scheme of the SU-8 moulding replica and the PDMS structures are presented in Figure 1.

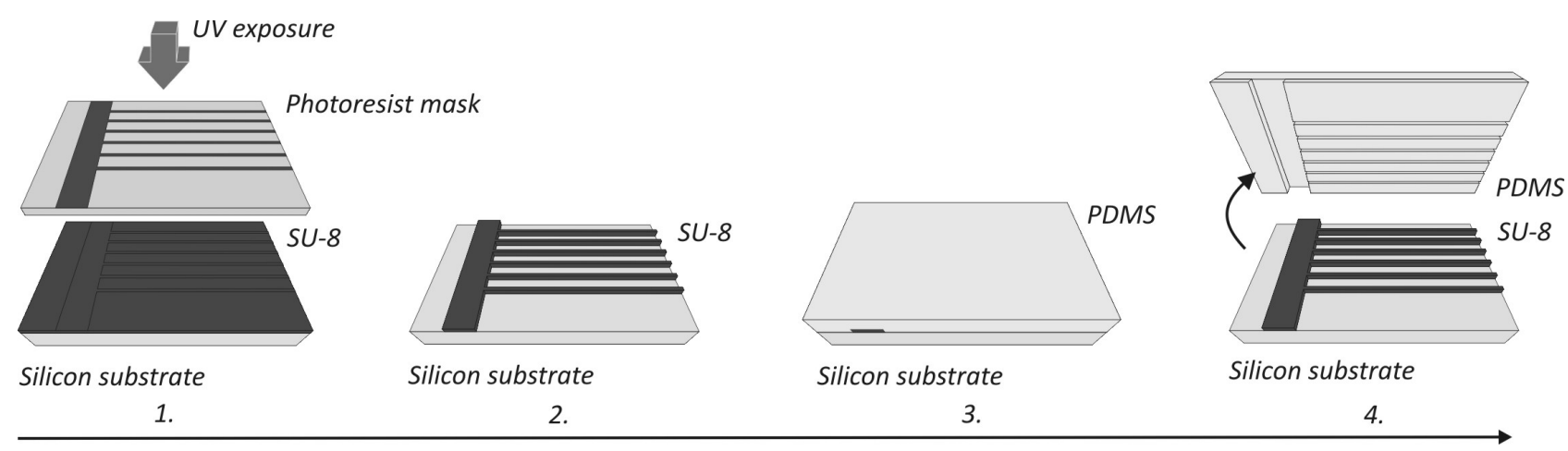

Fig. 1. Realisation steps of the microfluidic system in PDMS substrate applying SU-8 replica

Due to the low cost requirements of point-of-care applications, the photonic chip is fabricated from nano-composite polymeric materials, using highly scalable nano-imprint-lithography (NIL). The key issue investigated during the research regarding the integrability and final applicability of the developed structures is the appropriate polymer material selection, which has a natural impact on the leakage-free bonding between the optical (sensing) and the fluidic (sample transport) part of the device. To obtain a stable and high adhesion between the fluidic and the optical units, several different materials (polyimide, poly(methyl-metacrylate), SU-8) and bonding strategies were qualified. Adequate adhesion were achieved by subsequent silanisation processes (applying 
organofunctional alkoxysilanes e.g. APTES) and oxygen plasma treatments of polymer surfaces (see Figure 2).

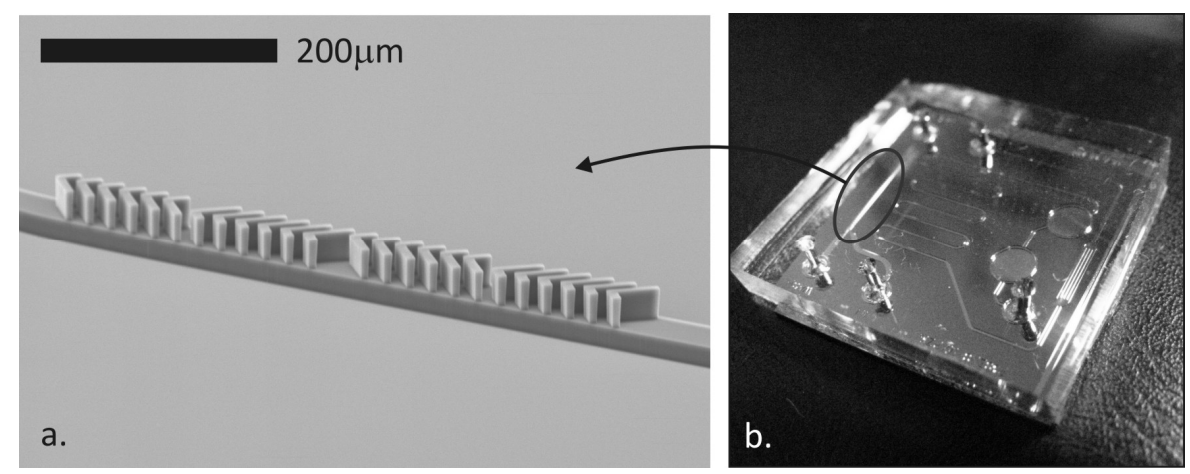

Fig. 2: Specific part (Herring-Bone type mixer structure) of the SU-8 moulding replica (a.) applied for the realisation of PDMS microfluidics (b.)

To study the surface behaviour of PDMS, a simple pair of microfluidic channel was realised using several composition of PDMS and Triton X-100 (TX-100) surfactant [10]. The adequate amount of surfactant was mixed into the raw polymer before polymerisation. Surfactant content was kept in the range $0.05-0.1-0.2 \%(\mathrm{v} / \mathrm{v})$. Polymerised substrates were sealed by glass utilizing oxygen plasma treatment.

\section{Hydrodynamic behaviour of modified PDMS microfluidic structure}

In our work, the influence of TX-100 on the surface wettability and the non-specific protein binding of the PDMS was studied in details. The change in wetting was characterised by dynamic contact angle measurement as presented in Figure 3.

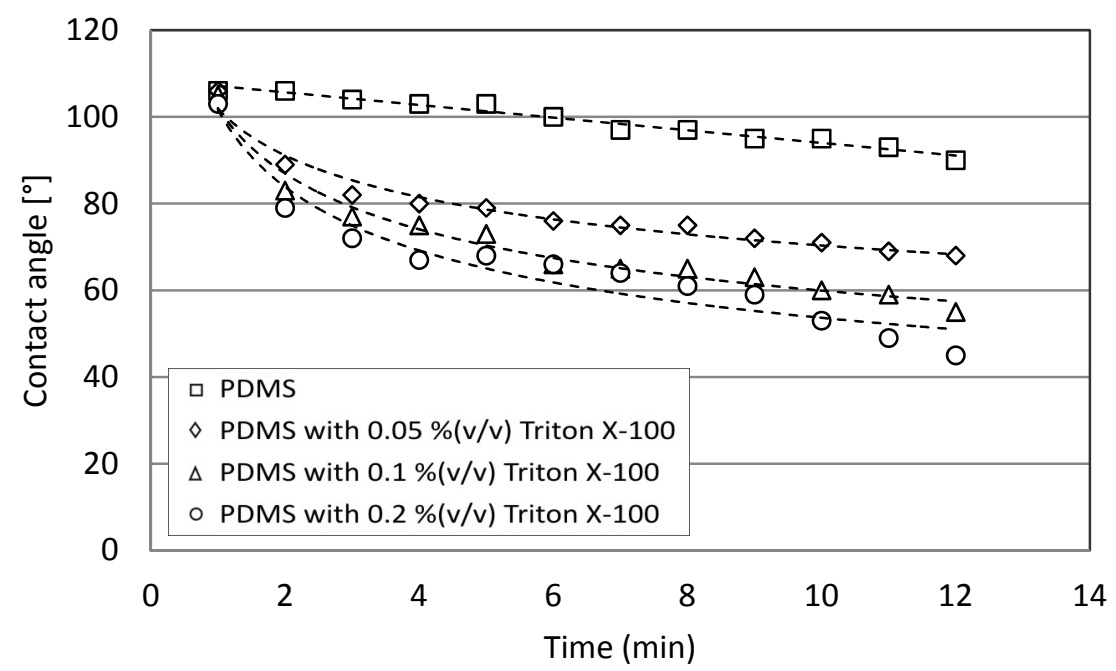

Fig. 3. Time dependent change of the surface contact angle of water (SACW) on different PDMS surfaces modified by the Triton X-100 surfactant

A significant change in the contact angle was observed, which is attributable to the embedded TX-100 molecules. Before ending in saturation, a rapid decreasing of the contact angle indicates the increasing surface concentration of the tenside molecules at the PDMS / water interface. The decrease in contact angles refer to the improvement of the wettability of PDMS surface, facilitating 
the design of microfluidics featured by enhanced capillarity. The characteristic capillary pressure in these systems, described by Equation 1, indicates a beneficial use in passive microfluidic pumps as well. The capillary pressure can be calculated as:

$$
P_{C}=-\gamma\left(\frac{\cos \alpha_{b}+\cos \alpha_{t}}{h}+\frac{\cos \alpha_{l}+\cos \alpha_{r}}{w}\right)
$$

where $\gamma$ denotes the surface tension at the fluid/gas interface, $\alpha_{b / t / l / r}$ are the contact angles of the fluid at the channel (bottom/top/left/right) walls, and $h$ and $w$ are the height and the width of the channel, respectively. Note, that the bottom channel wall was considered as glass.

Figure 4 represents the change of the PDMS surface from hydrophobic to hydrophilic (from positive to negative capillary pressure) in the case of different characteristic channel widths. The height of the microchannel was $20 \mu \mathrm{m}$, defined by the thickness of SU-8 2015 layer as moulding replica.

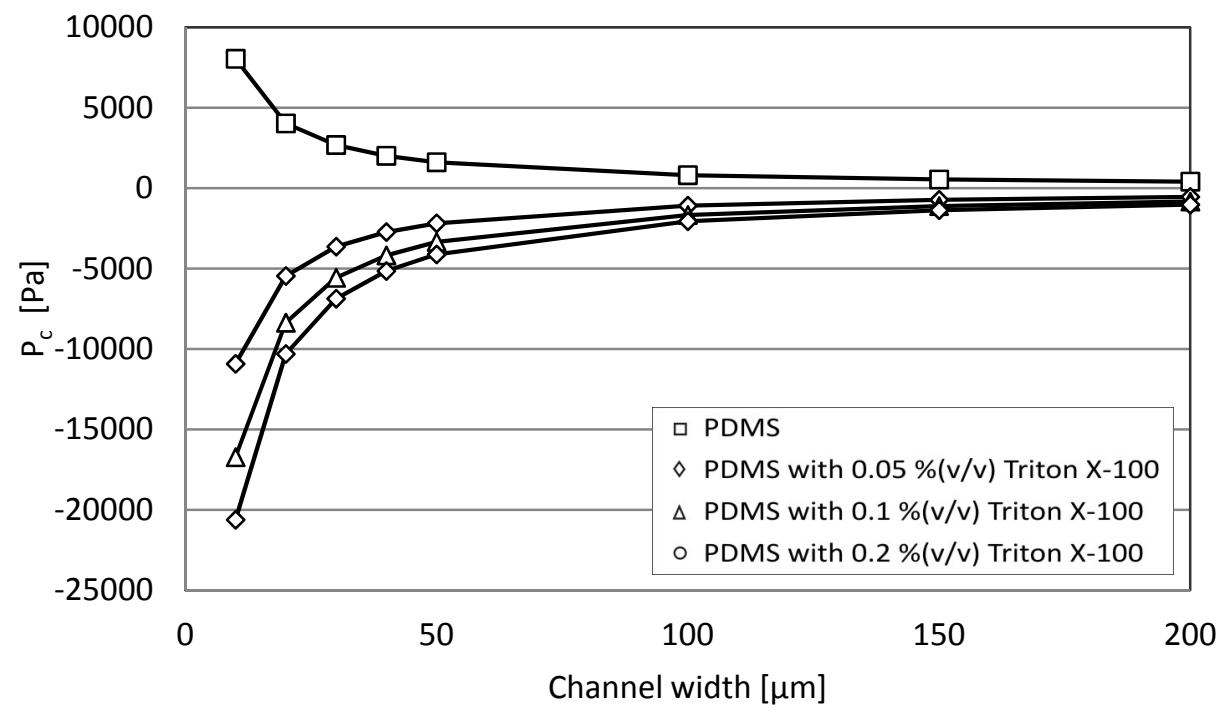

Fig. 4. Representative capillary pressures in the modified PDMS microchannels calculated from the measured contact angles

\section{Protein adsorption on channel sidewalls}

The non-specific adsorption of proteins on the PDMS surface is crucial regarding the applicability of the microfluidic structure in bioanalytical systems. Accordingly, the binding of proteins to the channel sidewalls was characterised in case of different material compositions. To qualify PDMS from this aspect, surface adsorption of fluorescein-isothiocyanate (FITC) labelled HSA was recorded by measuring the time dependent change of fluorescent intensity detected in the microchannel by using a fluorescent microscope. Various compositions of PDMS and alternative surface blocking method were also tested. In Figure 5, the irreversible protein adsorption on different PDMS surfaces was characterised by recording the relative fluorescent intensity of the applied HSA. The recorded relative values were determined as intensity increments compared to the natural intensity of the HSA solution. Fluorescent intensity of 100\% was detected, when PDMS surface was blocked by bovine serum albumin (BSA). The resident intensity after washing proves that irreversible HSA adsorption on the PDMS surface can be decreased by embedding TX-100 in the material. The effect of TX-100 concentration on the non-specific protein adsorption is summarised in Figure 6. 
We can conclude that protein adsorption can be decreased by almost $100 \%$ by this surface modification method, although the application of additional surface blocking protocols is advantageous in sensitive bioanalytical measurements alike.

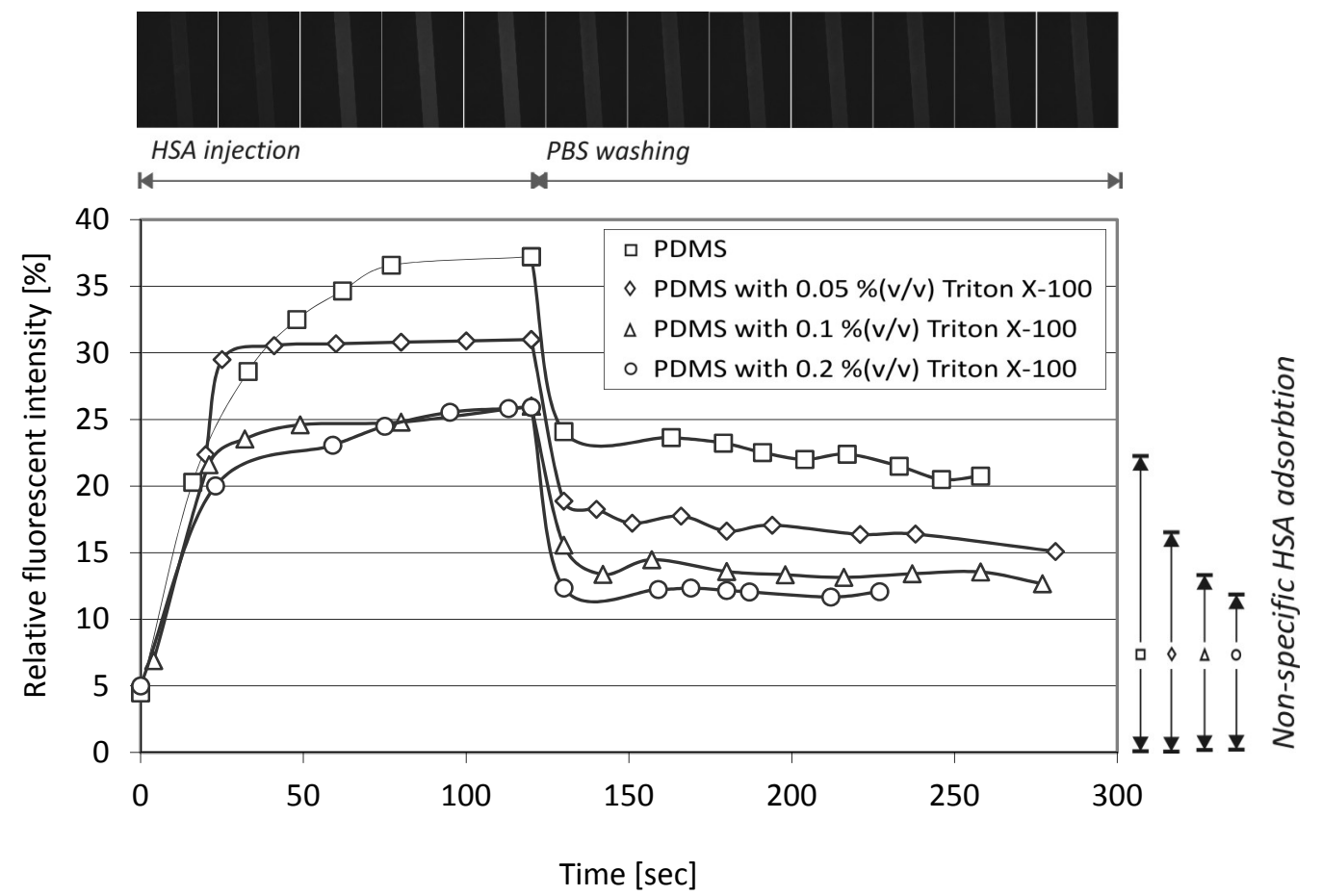

Fig. 5. Adsorption of the fluorescent labelled HSA protein on different PDMS surfaces characterised by time dependent fluorescent intensity. Over the diagram the sequence of the recorded fluorescent images represents the continuous change of the HSA concentration in the microfluidic channel during injection and washing periods.

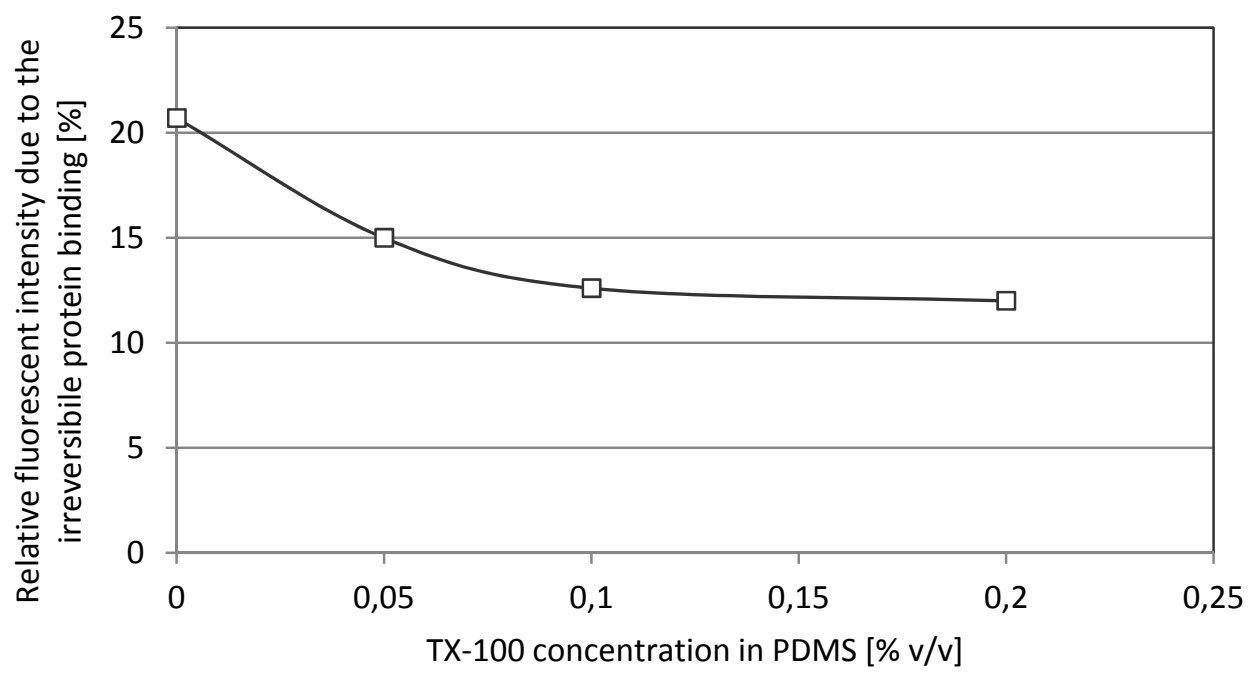

Fig. 6. The observed fluorescent intensities show the surface concentration of the HSA protein irreversible bonded at the channel surfaces as the function of the embedded TX-100 concentration. 


\section{Summary}

According to wettability and protein adsorption tests, surface characteristics of PDMS could be successfully modified by embedding TX-100 molecules into the bulk material. The hydrophobic behaviour of PDMS was altered by the admixture of tenside molecules in low concentration. Since the surface modification manner could be activated by contacting the material with water or water based solutions, technology for bonding PDMS to other substrates is not affected. The increase of TX-100 content in PDMS results in a significant decrease in contact angles, and therefore beneficially increases the capillary pressure in PDMS-based microfluidics like passive capillary pump systems.

Embedding of amphipathic molecules into the bulk PDMS phase has beneficial influence on the non-specific protein adsorption also. According to the results, the increasing surface concentration of TX-100 apparently decreased the quantity of proteins (HSA) irreversibly bonded onto the PDMS sidewalls. On the other hand, extremely sensitive bioanalytical measurements requires the application of additional blocking protocols. Further investigations are intended to characterise the long term stability of the modified PDMS, such as the surface and bulk diffusivity of the surfactant molecules.

\section{Acknowledgement}

The supports of the European Commission through the seventh framework program FP7-ICT4P3SENS (248304) and the János Bolyai fellowship of the Hungarian Academy of Sciences (recipient: Péter Fürjes) and the significant efforts of M. Erős and M. Payer in microtechnology are gratefully acknowledged.

\section{References}

[1.] A. Mata, A. J. Fleischman, and S. Roy, Characterization of polydimethylsiloxane (PDMS) properties for biomedical micro/nanosystems, Biomedical Microdevices 7 (4) (2005) 281-93

[2.] J. Zhou, A. V. Ellis, and N. H. Voelcker, Recent developments in PDMS surface modification for microfluidic devices, Electrophoresis 31 (1) (2010) 2-16

[3.] E. Delamarche, A. Bernard, H. Schmid, B. Michel, and H. Biebuyck, Patterned delivery of immunoglobins to surfaces using microfluidic networks, Science 286, (1997) 779-781

[4.] G. Sui, J. Wang, C.-C. Lee, W. Lu, S. P. Lee, J.V. Leyton, and A. M. Wu, Solution phase surface modification in intact PDMS microfluidic channels, Anal. Chem. 78 (2006) 55435551

[5.] www.harrickplasma.com/applications_microfluidic.php

[6.] J. Kim, M. K. Chaudhury, and M. J. Owen, The mechanism of hydrophobic recovery of PDMS elastomers exposed to partial electrical discharges, J. Colloid Interface Sci. 244 (2001) 200-207

[7.] S. Bhattacharya, Y. Gao, V. Korampally, M. T. Othman, S. A. Grant, K. Gangopadhyay, and S. Gangopadhyay, Mechanics of plasma exposed spin-on-glass (SOG) and polydimethyl siloxane (PDMS) surfaces and their impact on bond strength, Applied Surface Science 250 (2007) 4220-4225

[8.] S. Talaei, O. Frey, P. D. van der Wal, N. F. de Rooij, and M. Koudelka-Hep, Hybrid microfluidic cartridge formed by irreversible bonding of SU-8 and PDMS for multi-layer flow applications, Procedia Chemistry 1 (2009) 381-384

[9.] J. Seo, and L. P. Lee, Effects on wettability by surfactant accumulation/depletion in bulk polydimethylsiloxane (PDMS), Sensors and Actuators B 119 (2006) 192-198

[10.] www.sigmaaldrich.com (T8787 - CAS Number: 9002-93-1) 